\title{
Experimental time-domain study of THz signals from impulse excitation of a horizontal surface dipole
}

\author{
R. W. McGowan and D. Grischkowsky \\ School of Electrical and Computer Engineering and Center for Laser and Photonics Research, \\ Oklahoma State University, Stillwater, Oklahoma 74078
}

(Received 24 September 1998; accepted for publication 25 January 1999)

\begin{abstract}
Using optoelectronic techniques with sub-ps resolution, we have characterized the electric-field time-domain response from an impulsively excited, micron-sized dipole antenna on a dielectric surface. When detected by an adjacent dipole antenna $400 \mu \mathrm{m}$ distant, two primary signals are observed, a far-field pulse reflected from the back surface of the substrate and a surface-wave pulse. The surface-wave pulse appears as two distinct surface-propagating pulses despite originating from the dipole simultaneously. (C) 1999 American Institute of Physics. [S0003-6951(99)02712-6]
\end{abstract}

In the last decade there has been enormous progress in the generation, detection, and application of freely propagating short-pulse $\mathrm{THz}$ radiation. One proposed use for freely propagating $\mathrm{THz}$ waves is for on-chip or chip-to-chip communication of electrical signals. ${ }^{1,2}$ This approach could provide a method for overcoming the major absorption losses and dispersion that are incurred when ultrashort electrical pulses are propagated modest distances on conventional electrical wire interconnects. In this letter we present experimental results demonstrating the transmission and reception of ultrashort pulses of $\mathrm{THz}$ radiation between electrically isolated microstrip circuits along with a study of the mechanisms involved and show that there are two components to the detected signal, a far-field and a near-field signal. ${ }^{3}$ To gain an intuitive understanding of the characteristics of the detected signal, the transient electric field of a timedependent dipole moment in an isotropic media is discussed, as well as scaled comparisons of the $\mathrm{THz}$ experimental results to an analogous theoretical calculation in the field of electromagnetics at $\mathrm{GHz}$ frequencies. ${ }^{4}$ The experimental study also demonstrates the potential use of the far-field pulse for on-chip communication of picosecond pulses of $\mathrm{THz}$ radiation.

The study was performed with a dual dipole microcircuit on an ion-implanted silicon-on-sapphire (SOS) wafer with 80 $\mu \mathrm{m}$ dipoles separated by $400 \mu \mathrm{m}$, as shown in Fig. 1. Each coplanar strip line consists of two $5 \mu \mathrm{m}$ lines separated by 10 $\mu \mathrm{m}$ with the dipole antenna at the end of the strip line. For the transmitter, the strip line is biased with $5 \mathrm{~V}$; a $13 \mathrm{~mW}$ excitation beam of $60 \mathrm{fs}, 820 \mathrm{~nm}$, optical pulses from a Ti:sapphire mode-locked laser with a $100 \mathrm{MHz}$ repetition rate photoconductively shorts the lines at the antenna. This ultrafast process of shorting the transmitter dipole antenna in turn generates dipole radiation in the form of freely propagating $\mathrm{THz}$ waves. ${ }^{5}$ A second $13 \mathrm{~mW}$ detection beam of pulses from the same laser is focused at the dipole antenna gap on the receiver strip line. A current amplifier is connected across the receiver transmission lines, and lock-in detection techniques are incorporated. When an electric field is present across the receiver antenna coincident with a detection laser pulse, a dc current is detected which is proportional to the incident electric field. By scanning the relative delay of the optical detection beam to the arriving electric-field pulse, the pulse shape is obtained.

Due to the boundary conditions, the far-field $\mathrm{THz}$ radiation pulse from the dipole is emitted into a well-defined beam directed into the substrate. ${ }^{6-8}$ No far-field radiation is emitted along the surface and less than $1 \%$ of the radiation is emitted into the air normal to the surface. The far-field pulse is detected at the receiver dipole after it is internally reflected from the back of the $450 \mu \mathrm{m}$ thick SOS chip. From early experiments it became clear that the detected signal at the receiver antenna contained the superposition of both the reflected far-field and direct near-field components. To facilitate our understanding of these two field components of a dipole on a semi-infinite plane, it is advantageous to temporally separate the near- and freely propagating far-field components of the dipolar electric field. By contacting the SOS chip to a $3.2 \mathrm{~mm}$ thick $0^{\circ}$-cut sapphire plate, Fig. 1(b), the far-field pulse is delayed by an additional $65 \mathrm{ps}$ relative to the near-field pulse. Figure 2(a) shows an eight-scan average of the entire signal from this system. The data plot contains three distinct pulses, the near field (A), far field (B), and a small reflection of the far-field pulse from the boundary between the chip and plate $(\mathrm{C})$. The measured far-field pulse is nearly single cycle and has the same characteristics as the
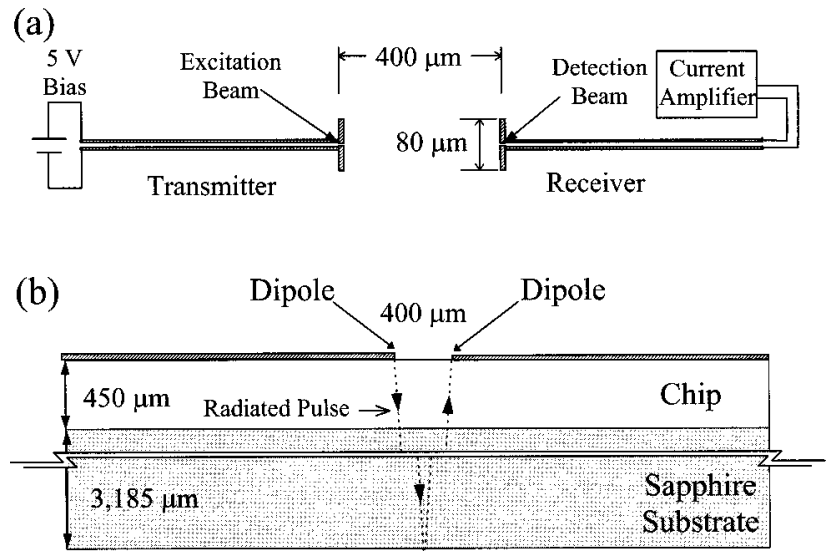

FIG. 1. (a) Diagram of the coplanar strip line dipole $\mathrm{THz}$ transmitter and receiver. (b) Cross-sectional view of the SOS chip contacted to a sapphire window showing radiated far-field $\mathrm{THz}$ pulse path. 

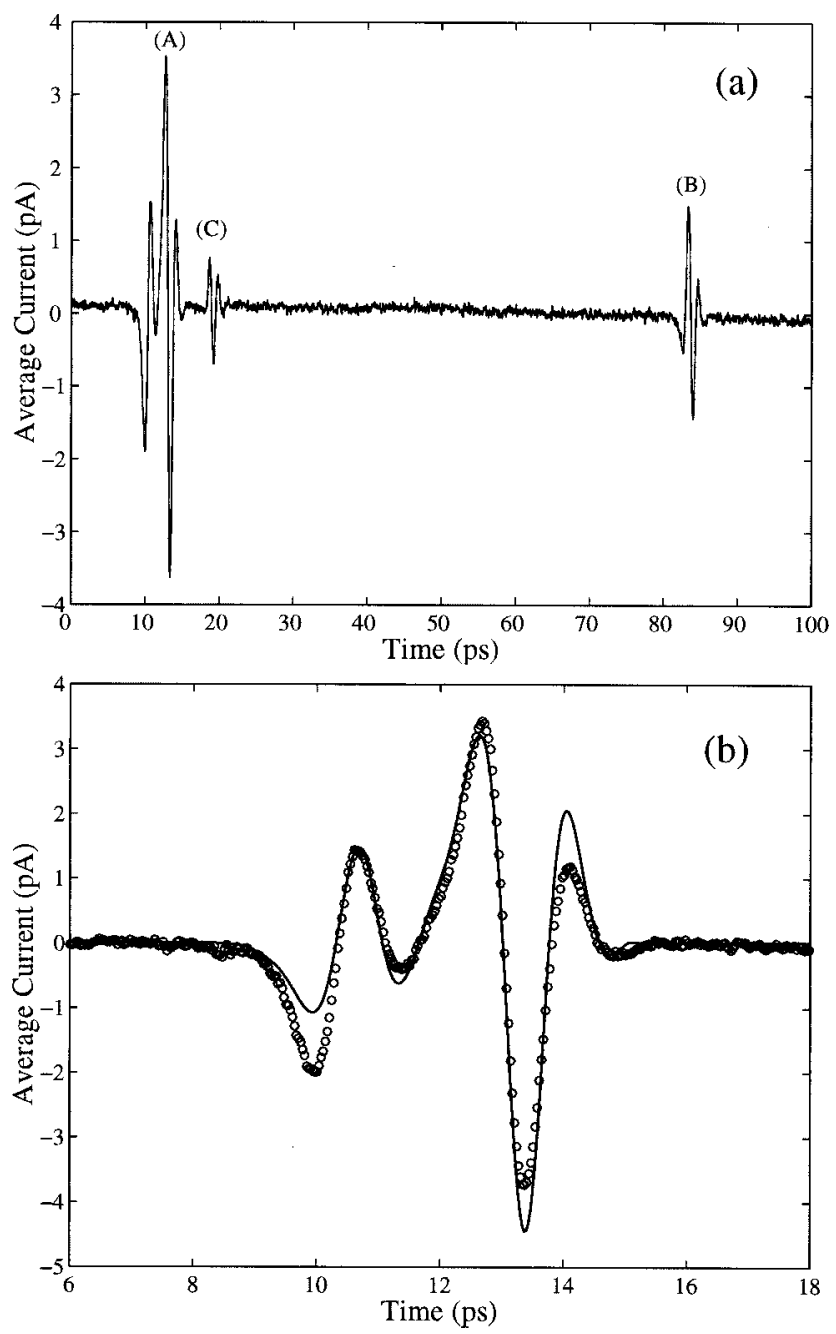

FIG. 2. Experimental results: (a) The three signals observed are (A) the near field, (B) freely propagating far field, and (C) a small reflection of the far-field pulse from the chip-sapphire substrate boundary. (b) Plot of the observed near-field pulse (A), circles, with the modeled fit, solid line, overlaid.

typical freely propagating $\mathrm{THz}$ pulse generated by photoconductive antennas. ${ }^{9}$ The far-field pulse has a 0.47 ps full width at half maximum (FWHM) pulse width with a measured frequency bandwidth of $0.6 \mathrm{THz}$ FWHM centered at $0.7 \mathrm{THz}$. Some reshaping of the far-field pulse is expected due to the index of the sapphire dispersion. The near-field pulse, however, contains a complex structure of which the mechanism is not easily understood. It is clear from the timing issues that the detected pulse (A) does not propagate through the substrate and reflect from the backside of the sapphire plate, like (B), or the boundary layer as pulse (C), rather it must travel along the air-SOS boundary where it is detected by the receiver antenna.

To gain an intuitive understanding of the detected signal, one can consider the electric field from a Hertzian dipole in an isotropic media, with refractive index $n=\sqrt{\epsilon / \epsilon_{0}}$. The electric field written in terms of the time-dependent dipole moment $p(t)$ is ${ }^{1,10}$

$$
E_{\theta}(r, \theta, t)=\frac{\sin \theta}{4 \pi \epsilon}\left(\frac{1}{r^{3}} p(t)+\frac{n}{c r^{2}} \dot{p}(t)+\frac{n^{2}}{c^{2} r} \ddot{p}(t)\right) .
$$

Here, $r$ is the distance from the dipole; $\theta$ is the angle relative to the dipole axis, which is $90^{\circ}$ in our case; and $c$ is the speed of light in vacuum. The three components to this field are, from left to right: the quasistatic field, the near field, and the far field. ${ }^{3}$ It is immediately noticed from this equation that the near-field component is one order lower in the time derivative than the far-field component. The data yield a wellseparated far-field pulse which can give insight into the structure of the near-field component. Assuming pulse (A) in Fig. 2(a) is a near-field signal, one expects the shape to be the time integral of the far-field signal (B), which clearly is not the case.

Electromagnetic theoretical calculations have been performed for nanosecond current impulse excitations of a horizontal Hertzian dipole on a multilayered dielectric medium. ${ }^{4}$ Although the time and size scale of the theoretical calculations are a factor of 1000 longer than in our experiment, there is a direct correspondence of these $\mathrm{GHz}$ microwave predictions and our $\mathrm{THz}$ measurements because for equivalent dielectric properties and similar wavelength/size ratios there is a one-to-one correspondence of the results. Contrary to the known far-field radiation pattern, which does not allow surface propagation, ${ }^{6-8}$ for near-field radiation the theory predicts the existence of two "direct" surface pulses as well as the shape of these pulses being the time integral of the far-field "reflected" pulses. To verify that the experimentally detected near-field pulse is indeed the superposition of the predicted two "direct" pulses, a fit was performed on the actual near-field signal using two pulses which have the shape of the time integral of the observed far-field pulse. The later time pulse used in the empirical fit is inverted, scaled by 3.0, shifted by $2.70 \mathrm{ps}$, and superimposed with the earlier time pulse. Since the dispersion of sapphire is well known, the pulse reshaping due to the propagation through the sapphire substrate was removed prior to integrating the far-field pulse. The fit of the near-field signal is shown in Fig. 2(b) as the solid line. All of the characteristics and main features are mimicked well by the fit, and thereby confirm our observation of the two "direct" pulses of Ref. 4.

In our experimental arrangement the receiving dipole which is also on the surface has the ability to measure electromagnetic disturbances that propagate both in the dielectric and in the air. The two observed components [Fig. 2(a), (A)], which have separated in time due to their different propagation velocities, are the "direct" near-field components of the impulse dipole. The first near-field pulse propagates the 400 $\mu \mathrm{m}$ distance to the antenna along the surface through air and the second pulse, which is emitted simultaneously, propagates the same distance, but through the sapphire substrate $\left(n_{0}=3.07\right)$. The separation between arrival times of 2.76 $\pm 0.06 \mathrm{ps}$ is expected, which is in agreement with the required fit parameter of $2.70 \mathrm{ps}$. In addition, from the high temporal precision of our data and precise measurements of the sapphire plate thickness $(3.185 \pm 4 \mu \mathrm{m})$ and chip thickness $(450 \pm 2 \mu \mathrm{m})$, the dipole excitation time $(t=0)$ is calculated. The propagation time for the far-field pulse is calculated to be $74.50 \pm 0.15 \mathrm{ps}$, subtracting this from the farfield pulse center position of $83.34 \pm 0.05 \mathrm{ps}$, the $t=0$ time position of $8.84 \pm 0.16 \mathrm{ps}$ is obtained. From the $t=0$ point on the data scan, the velocities of the two near-field pulses are calculated and the effective indices of refraction obtained. 
For the first arriving near-field pulse a propagation speed of $0.92 c \pm 0.10 c$ is measured, which yields an index of refraction of $n_{1}=1.09 \pm 0.12$ and for the second arriving pulse the speed is $0.32 c \pm 0.01 c$ with a corresponding index of refraction of $n_{2}=3.13 \pm 0.13$. The calculated index of refraction for the first and second pulse correlate well with that of air (1.00) and the ordinary index of sapphire (3.07), respectively, thereby, confirming the predicted propagation speeds. ${ }^{4}$ Also, with a ps impulse excitation of a short dipole, Eq. (1) shows that for a homogeneous dielectric, starting at a distance of about $100 \mu \mathrm{m}$ in sapphire, the far-field term dominates. Yet, for the case of a dipole on a dielectric surface the smaller near-field term is observed in complete isolation, since there is no far-field propagation along the surface due to the boundary conditions. ${ }^{6-8}$

Although the time and size scale of the previous theoretical $\mathrm{GHz}$ electromagnetic calculations are 1000 times longer than in this $\mathrm{THz}$ experiment, our scaled results confirm many of the observations of the theory. ${ }^{4}$ The calculation predicts back- "reflected" waves as well as the two "direct" waves, one in the substrate and one above the substrate, which is precisely what we experimentally observe. In the theory, these two direct waves exhibit several features: they decay as $1 / r^{2}$, as is expected for the near-field component; they have a relative inversion to each other, the magnitude of the dielectric signal is several times larger than the signal through air; and they have a shape which is a time integral of the far-field back-reflected pulse. All these features are precisely confirmed with the experimental results presented here. Due to the geometry of our experiment, there is one class of waves defined in the theoretical calculation called the "lateral", waves, which are not observed.

The relative magnitude of the near- and far-field pulses obtained from Eq. (1) for the Hertzian dipole can be compared with the experimental results. In the calculation, a Gaussian dipole pulse with $1 / e$ full width of 1.2 ps is used. The width and shape of the dipole pulse were determined by fitting the second numerical time integral of the observed far-field pulse. For the pulses in the dielectric, the ratio of the maximum of the near-field term at $400 \mu \mathrm{m}$ to the maximum of the far-field term at $7280 \mu \mathrm{m}$ is evaluated from Eq. (1) to be 1.14. To determine the corresponding ratio from the experimental data, the larger peak of the near-field pulse (A) is compared to the far-field pulse (B), accounting for the partial reflection of 0.51 of the sapphire plate. The experimentally obtained ratio is 1.17 , which compares very well with the calculated value.

In conclusion, via a simple two-dipole antenna system on a sapphire substrate we have identified two main features from the study of on-chip communication of $\mathrm{THz}$ pulses. The freely propagating, far-field $\mathrm{THz}$ signal with its clean bipolar characteristic and sub-ps pulse width is well suited for high-frequency on-chip communication or synchronous triggering of electrically isolated circuits as well as interchip communication. A second signal pulse is detected which is due to the near-field component and consists of two distinct pulses. These two near-field pulses have the same pulse shape, the time integral of the far-field radiation pulse; one pulse is inverted with respect to the other. It is observed that the $\mathrm{THz}$ experimental results scale and compare well with a $\mathrm{GHz}$ electromagnetic theoretical calculation of a horizontal dipole on a dielectric, excited by an electrical impulse 1000 times longer than that used in this experiment. This correspondence suggests that macroscopic geological problems in the $\mathrm{GHz}$ regime could be studied by our techniques, if modeled appropriately on the laboratory scale. It is also concluded that the range and characteristics of the near-field pulse are important issues which need to be considered when designing any ultrashort electrical pulse systems as well as in the design of ultrafast on-chip communication architectures.

This work was supported in part by the National Science Foundation and the Army Research Office.

${ }^{1}$ D. H. Auston, K. P. Cheung, and P. R. Smith, Appl. Phys. Lett. 45, 284 (1984)

${ }^{2}$ I. N. Duling III, D. Grischkowsky, J.-M. Halbout, and M. B. Ketchen, U.S. Patent No. 5,056,111 (1991).

${ }^{3}$ J. D. Kraus, Antennas, 2nd ed. (McGraw-Hill, New York, 1988), p. 60.

${ }^{4}$ R. Dai and C. T. Young, IEEE Trans. Antennas Propag. 45, 1023 (1997).

${ }^{5}$ M. van Exter, Ch. Fattinger, and D. Grischkowsky, Appl. Phys. Lett. 55, 337 (1989).

${ }^{6}$ W. Lukosz, J. Opt. Soc. Am. 69, 1495 (1979).

${ }^{7}$ N. Engheta and C. H. Papas, Radio Sci. 17, 1557 (1982).

${ }^{8} \mathrm{Ch}$. Fattinger and D. Grischkowsky, in Proceedings of the Picosecond Electronics and Optoelectronics Topical Meeting (Salt Lake City, UT), March 8-10, 1989, p. 225.

${ }^{9}$ M. van Exter and D. Grischkowsky, IEEE Trans. Microwave Theory Tech. 38, 1684 (1990).

${ }^{10}$ The time-dependent equation is obtained by taking a Fourier transform of the commonly derived frequency-dependent equation for the $E$ field of a Hertzian dipole, for example, J. D. Kraus, Antennas, 2nd ed. (McGrawHill, New York, 1988), p. 207, Eq. (39). 